\title{
SUSPEITA DA PERDA AUDITIVA POR FAMILIARES
}

\author{
Hearing loss suspected by the family
}

\author{
Claudete Ferreira de Souza Monteiro ${ }^{(1)}$, Janaína Mendes de Sousa Caldas ${ }^{(2)}$, \\ Nilena Carla Monteiro Araújo Arêa Leão ${ }^{(3)}$, Marina Rocha Soares ${ }^{(4)}$
}

\begin{abstract}
RESUMO
Objetivos: caracterizar e analisar a suspeita de perda auditiva, por parte de familiares, em crianças assistidas na Associação de Pais e Amigos do Deficiente Auditivo (APADA) em Teresina-PI. Métodos: estudo descritivo realizado na APADA no período de setembro a outubro de 2006, com aplicação de formulários para 14 mães e/ou responsáveis para levantamento das variáveis: identificar o familiar que suspeitou da perda auditiva na criança; o motivo que levou a suspeita; a idade da criança quando da detecção pelo familiar; a atitude da família após a descoberta da perda auditiva; a idade da criança quando ocorreu procura profissional e a orientação recebida; o tempo entre a suspeita da perda auditiva, por parte do familiar, e a confirmação profissional de diagnóstico. Resultados: os dados apontam que $86 \%$ da suspeita foi percebido pelas mães. O motivo da suspeita foi pela não reação ao som por parte da criança (71\%). 36\% dos familiares perceberam o problema na idade de 7 a 12 meses. $79 \%$ tiveram como primeira atitude procurar o médico, 36\% procuraram o profissional da saúde com até um ano de idade, $29 \%$ receberam a orientação do profissional para o uso do aparelho auditivo e tiveram a confirmação da perda auditiva de 7 meses a 1 ano de idade ou após os 4 anos. Conclusão: o diagnóstico da perda auditiva foi tardio frente a suspeita por parte dos familiares e dos profissionais de saúde e não estiveram relacionadas com os programas da triagem auditiva neonatal.
\end{abstract}

DESCRITORES: Audição; Perda Auditiva; Linguagem; Diagnóstico

\section{INTRODUÇÃO}

O ouvido é um dos órgãos que merece atenção no processo de desenvolvimento infantil. $O$ sis-

(1) Enfermeira; Docente da Faculdade de Ciências Humanas e Tecnológicas do Piauí e da Universidade Federal do Piauí, UFPI, Teresina, PI; Coordenadora do Programa de Mestrado em Enfermagem da Universidade Federal do Piauí, UFPI, Teresina, PI; Subcoordenadora do Núcleo de Estudos sobre Mulher e Relações de Gênero da Universidade Federal do Piauí, UFPI, Teresina, PI; Doutora em Enfermagem.

(2) Fonoaudióloga; Clínica Desenvolver na Maternidade Santa Fé, Teresina, Pl; Pós-graduanda em Audiologia Clínica pela Faculdade de Ciências Humanas e Tecnológicas do Piauí.

(3) Fonoaudióloga; Clínica Desenvolver da Maternidade Santa Fé, Teresina, PI; Pós-graduanda em Linguagem pela Faculdade de Ciências Humanas e Tecnológicas do Piauí.

(4) Fonoaudióloga; Clínica Flávio Santos e da Fundação de Seguridade Social, GEAP, Teresina, PI; Docente das disciplinas de Audiologia Educacional e Estágio Supervisionado em Audiologia Educacional I e II, da Faculdade de Saúde, Ciências Humanas e Tecnológicas do Piauí, NOVAFAPI, Teresina, PI; Especialista em Audiologia e Equilibriometria Clínica pela Universidade Federal de Pernambuco.

Conflito de interesse: inexistente tema auditivo do ser humano é capaz de detectar os sons produzidos pelo falante e, assim, construir o domínio da linguagem. Essa linguagem é importante para a comunicação entre os seres humanos. $A$ audição constitui-se em um pré-requisito para a aquisição e o desenvolvimento da linguagem. A audição e linguagem são funções correlacionadas e interdependentes ${ }^{1}$.

A perda auditiva na infância é um grave problema de saúde pública, tanto pela frequência, como pelos intensos prejuízos linguísticos, educacionais e psicossociais que pode determinar ${ }^{2}$. A relação existente entre a audição e o desenvolvimento da linguagem no ser humano torna o diagnóstico da deficiência auditiva de extrema importância. Assim, o diagnóstico precoce em crianças é altamente desejável, nos primeiros seis meses, já que as deficiências auditivas podem, em longo prazo, levar a alterações irreversíveis da aquisição de linguagem e da habilidade cognitiva ${ }^{3}$.

Uma criança em cada 1000 nasce com perda auditiva profunda. Além disso, duas crianças em 1000 adquirem a deficiência auditiva na infância. No caso de crianças que possuem fatores de riscos 
para a deficiência auditiva, esta proporção aumenta para 1 em cada 50 nascidas vivas ${ }^{4}$.

A incidência da surdez nas maternidades é superior a outras patologias avaliadas em triagem, comparando com outras doenças de triagem universal, como fenilcetonúria $(0,07 / 1.000)$, hipotireoidismo congênito $(0,17 / 1.000)$ e anemia falciforme $(0,20 / 1.000)$, dispomos de justificativas para o estabelecimento de um programa de triagem auditiva ${ }^{5}$.

A importância dos primeiros anos de vida para o desenvolvimento da criança e especialmente da linguagem exige que o diagnóstico da deficiência de audição seja realizado o mais cedo possível, preferencialmente antes dos seis meses de vida ${ }^{6}$.

As principais causas da perda auditiva para a criança podem ser verificadas no atraso do desenvolvimento da capacidade de comunicação receptiva e expressiva (fala e linguagem); na dificuldade de linguagem que causa problemas de aprendizagem, reduzindo o desempenho acadêmico; na dificuldade de comunicação que muitas vezes induz o isolamento social e a baixa-estima; e no impacto nas escolhas vocacionais ${ }^{7}$.

Pesquisa entrevistando 30 pediatras demonstra que eles têm conhecimento sobre a atuação fonoaudiológica em relação às diferentes áreas como fala $(100 \%)$, linguagem oral $(90 \%)$, voz $(90 \%)$ e motricidade oral $(86,6 \%)$, porém revelam um desconhecimento por parte de alguns pediatras em relação a algumas áreas de atuação como audição $(80 \%)$ e linguagem escrita $(50 \%)^{8}$.

Diante da problemática apresentada realizou-se este estudo com o objetivo de analisar as características da suspeita da perda auditiva, por parte de familiares, em crianças assistidas na Associação de Pais e Amigos do Deficiente Auditivo (APADA) em Teresina - Piauí (PI).

\section{MÉTODOS}

Esta pesquisa foi delineada como estudo exploratório de natureza descritiva com abordagem quantitativa.

O local da pesquisa foi a APADA em Teresina - PI. Esta organização é caracterizada como nãogovernamental - ONG, filiada a Federação Nacional das APADAS. Instituição de Utilidade Pública Municipal - Lei $n^{\circ} 2.526$ e de Utilidade Pública estadual - Lei $n^{\circ}$ 1197. A APADA foi criada em 30/11/1991, por um grupo de pais de deficientes auditivos e por profissionais engajados na luta por melhores condições de vida destas pessoas.

Constituíram sujeitos do estudo, 14 mães ou responsáveis pelos alunos assistidos na APADA, em Teresina - PI. Os sujeitos convidados assinaram um Termo de Consentimento Livre e Esclarecido autorizando a participação na pesquisa, conforme a Resolução 196/96, que trata de pesquisa envolvendo seres humanos.

Para registro dos dados utilizou-se um formulário estruturado, pelas autoras da pesquisa, com perguntas fechadas e abertas para levantamento das variáveis: identificar o familiar que suspeitou da perda auditiva na criança; o motivo que levou a suspeita; a idade da criança quando da detecção pelo familiar; a atitude da família após a descoberta da perda auditiva; a idade da criança quando ocorreu procura profissional e a orientação recebida; o tempo entre a suspeita da perda auditiva, por parte do familiar, e a confirmação profissional de diagnóstico.

Os formulários foram aplicados pelas autoras do trabalho, no período de setembro a outubro de 2006 (Figura 1). Cada entrevista durou cerca de 10 minutos.

Esta pesquisa foi aprovada pelo Comitê de Ética em Pesquisa da Faculdade NOVAFAPI, sob no 1448/06 e obteve o consentimento da instituição onde foram coletados os dados.

A análise é do tipo descritivo e apresentado em tabelas.

\section{RESULTADOS}

Os laudos audiológicos, nos exames disponíveis das crianças assistidas na associação, revelam 14 (100\%) perda auditiva sensorioneural bilateral com um $(7,14 \%)$ sujeito de grau variando entre moderada à severa, $11(78,57 \%)$ sujeitos com grau de severa à profunda e dois $(14,29 \%)$ de grau profunda. $14(100 \%)$ das crianças não utilizam o aparelho auditivo bilateral.

Dividiu-se a faixa etária dos indivíduos em intervalos de cinco anos. Há predominância do grupo etário de cinco $(35,71 \%)$ indivíduos de 11 a 15 anos. Consta também um $(7,14 \%)$ indivíduo de 1 a 5 anos, três $(21,43 \%)$ de 6 a 10 anos, três $(21,43 \%)$ 16 a 20 anos, e dois (14,29\%) de 21 a 25 anos. Em relação ao sexo dos sujeitos do estudo, quatro $(28,57 \%)$ foram do sexo feminino e $10(71,43 \%)$ do sexo masculino.

Em relação ao grau de parentesco com o indivíduo, verificou-se que os entrevistados foram assim representados: $10(71,43 \%)$ mães, uma $(7,14 \%)$ irmã, uma $(7,14 \%)$ mãe adotiva e duas $(14,29 \%)$ tias.

Verificou-se qual membro da família percebeu o problema da audição da criança. Os dados mostram a prevalência de $12(85,72 \%)$ mães, seguido de uma $(7,14 \%)$ tia e um $(7,14 \%)$ avô.

Quanto ao motivo que levou o familiar a suspeitar do problema auditivo na criança, o estudo mostra que $10(71,43 \%)$ suspeitaram porque a criança não reagia na presença de qualquer barulho, três 


\section{FORMULÁRIO}

Caracterização do sujeito:

Genêro ( ) masculino ( ) feminino

Idade: anos

I) Quem percebeu o problema de audição da criança?

1. Mãe / pai

2. Amigos

3. Familiares (tios, irmãos, outros)

II) Qual o motivo que fez você suspeitar desse problema auditivo?

III) Qual a idade da criança quando foi detectado o problema auditivo?
1. Até 3 meses
2. 4 meses a 6 meses
3. 7 meses a 12 meses
4. 13 meses a 3 anos
5. Após os 3 anos

IV) Qual sua primeira atitude depois de perceber o problema na audição?

V) Quando procurou pela primeira vez um profissional, da área da saúde, para tratar a audição da criança?

1. De 0 a 6 meses

2. De 7 a 12 meses

3. Após 12 meses

4. Após 2 anos

5. Após 5 anos

VI) Qual a orientação recebida do profissional?

VII) Qual a idade da criança quando ocorreu a confirmação profissional da perda auditiva, através do exame?
1. De 0 a 6 meses
2. 7 meses a 1 ano
3. 1 ano a 2 anos
4. 2 anos aos 3 anos
5. Após os 3 anos
6. Após os 4 anos

\section{Figura 1 - Questionário utilizado}

$(21,43 \%)$ quando a criança não atendeu à voz do familiar e um $(7,14 \%)$ suspeitou após uma gripe que a criança teve, conforme a Tabela 1.

Pesquisando a idade da criança quando da suspeita de perda auditiva por familiares da criança, três $(21,43 \%)$ suspeitaram de 0 a 3 meses de vida, três $(21,43 \%)$ dos 4 aos 6 meses, cinco $(35,71 \%)$ detectou entre os 7 aos 12 meses, dois (14,29\%) de 13 meses aos 3 anos e um (7,14\%) após os três anos, conforme a Tabela 2. 
Tabela 1 - Motivo que levaram a suspeita do problema auditivo na criança

\begin{tabular}{lccc}
\hline & & № & $\%$ \\
\hline \multirow{3}{*}{ Motivo } & Não reagia ao barulho & 10 & 71.43 \\
& Não atendia a voz & 3 & 21.43 \\
& Após uma gripe & 1 & 7.14 \\
\hline Total & & 14 & 100.00 \\
\hline
\end{tabular}

Tabela 2 - Idade da criança quando o familiar suspeitou do problema auditivo

\begin{tabular}{lccc}
\hline & & № & $\%$ \\
\hline \multirow{3}{*}{ Faixa etária } & De 0 a 3 meses & 3 & 21.43 \\
& De 4 a 6 meses & 3 & 21.43 \\
& De 7 a 12 meses & 5 & 35.71 \\
& De 13 meses a 3 anos & 2 & 14.29 \\
\hline Total & Após 3 anos & 1 & 7.14 \\
\hline
\end{tabular}

Em relação à atitude do entrevistado frente à suspeita de deficiência auditiva, verificou-se que $11(78,58 \%)$ procuraram um profissional médico. Outras atitudes como: um $(7,14 \%)$ para a procura de meios para realizar o exame de audição, um (7,14\%) para o abandono da criança, e um $(7,14 \%)$ na atitude de levar ao rezador, também se fizeram presente na pesquisa.

Quanto à idade da criança, relatada pelo familiar ao buscar um profissional da área de saúde, o estudo mostra que dois $(14,29 \%)$ procuraram o profissional de 0 a 6 meses de idade, cinco (35,71\%) após levantamento da suspeita entre 7 e 12 meses, dois $(14,29 \%)$ após os 12 meses, dois $(14,29 \%)$ após 2 anos e três $(21,43 \%)$ após os 5 anos. Na amostragem percebeu-se que cinco $(35,71 \%)$ dos entrevistados procuraram tratamento auditivo quando a criança estava entre o primeiro ano de vida, sendo que uma prevalência de três $(21,43 \%)$ procuraram somente após os 5 anos, conforme exposto na Tabela 3.
Na Tabela 4 podem-se visualizar os dados referentes à atitude dos profissionais de saúde frente à queixa apresentada pelo familiar. Houve predomínio de quatro $(28,57 \%)$ para indicação do uso do aparelho auditivo; um $(7,14 \%)$ encaminhamento para a avaliação fonoaudiológica; três $(21,43 \%)$ relatos de orientações para encaminhamentos a exames auditivos; uma $(7,14 \%)$ indicação do uso de aparelho e terapia concomitantemente; e três $(21,43 \%)$ revelação da possibilidade de surdez na criança. Também foi referido pelos profissionais duas $(14,29 \%)$ orientações de remarcar nova avaliação.

Com relação ao tempo em que ocorreu a confirmação profissional da perda auditiva, feita através de exames, um $(7,14 \%)$ sujeito relata que ocorreu em até 6 meses, quatro (28,57\%), entre 7 meses a 1 ano, dois (14,29\%), de 1 a 2 anos, três $(21,43 \%)$ de 2 a 3 anos e quatro $(28,57 \%)$ após os 4 anos, conforme exposto na Tabela 5.

Ao relacionar o tempo entre a suspeita do familiar e a confirmação profissional da perda auditiva,

Tabela 3 - Idade da criança em que ocorreu a procura por um profissional da área da saúde

\begin{tabular}{lccc}
\hline & & № & $\%$ \\
\hline \multirow{4}{*}{ Faixa etária } & De 0 a 6 meses & 2 & 14.29 \\
& De 7 a 12 meses & 5 & 35.71 \\
& De 12 meses a 2 anos & 2 & 14.29 \\
& De 2 a 5 anos & 2 & 14.29 \\
\hline Total & Após 5 anos & 3 & 21.43 \\
\hline
\end{tabular}


Tabela 4 - Orientação dada pelo profissional procurado

\begin{tabular}{lccc}
\hline & № & $\%$ \\
\hline Orientação & 3 & 21.43 \\
$\begin{array}{c}\text { Encaminhou para } \\
\text { exames auditivos } \\
\text { Solicitou espera para } \\
\text { outra avaliação } \\
\text { Encaminhou para } \\
\text { Fonoaudiologia } \\
\text { Indicou o uso de } \\
\text { aparelho } \\
\text { Indicou o uso de } \\
\text { aparelho e terapia } \\
\text { fonoaudiológica } \\
\text { Revelou possibilidade } \\
\text { de surdez }\end{array}$ & 2 & 14.29 \\
\hline Total & 4 & 7.14 \\
\hline
\end{tabular}

Tabela 5 - Tempo entre a suspeita do familiar e a confirmação profissional da perda auditiva

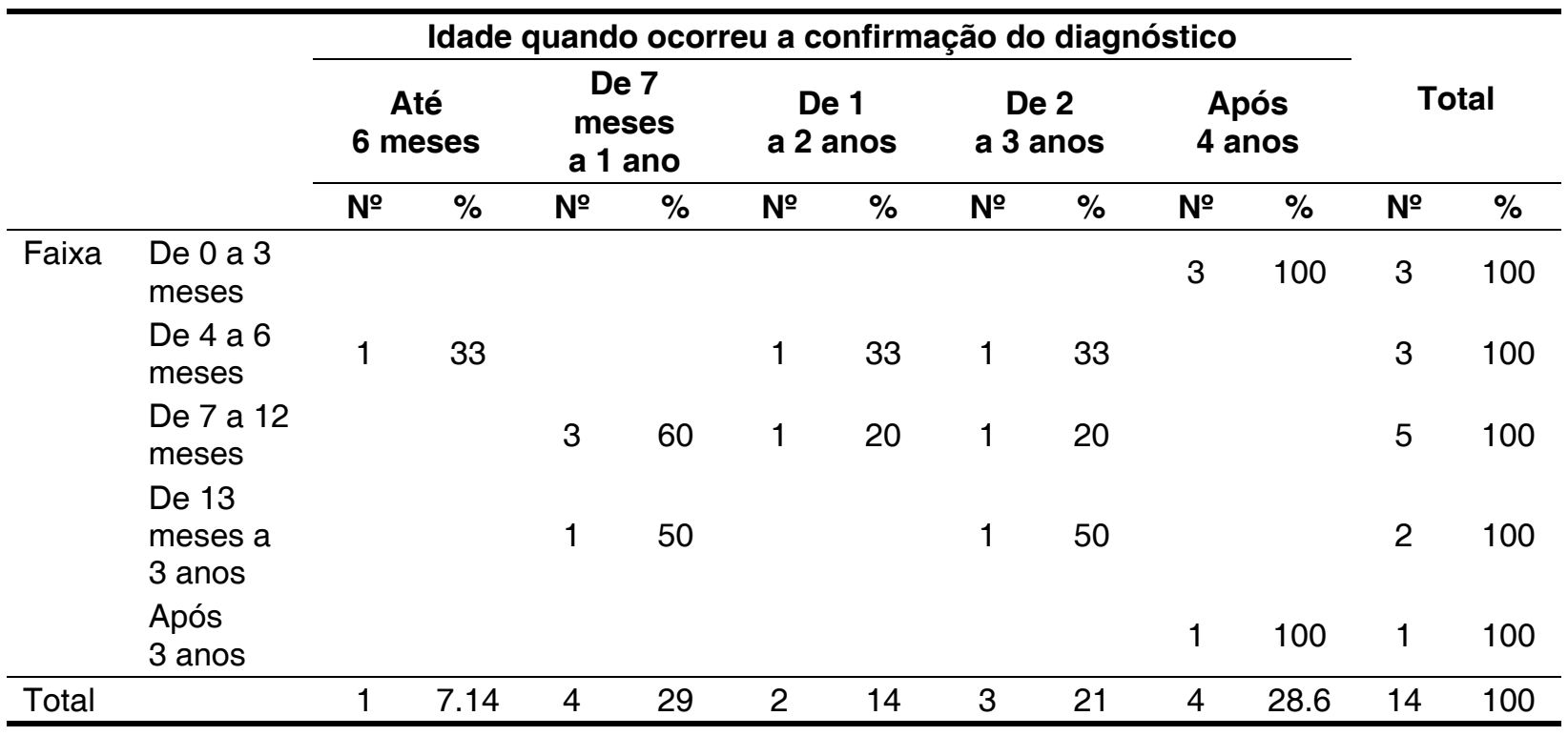

constatou-se que os familiares que suspeitaram da perda auditiva até os 3 meses de idade da criança, só tiveram confirmação após os 4 anos. Dos que suspeitaram entre 4 a 6 meses, um (33\%) teve confirmação até 6 meses, um (33\%) entre 1 e 2 anos e um $(33 \%)$ entre 2 e 3 anos. Para a suspeita entre 7 a 12 meses, a ocorrência da confirmação foi de três $(60 \%)$ entre os 7 meses a 1 ano, uma (20\%) entre 1 a 2 anos, e uma (20\%) e entre 2 a 3 anos. Os familiares que suspeitaram entre 13 meses a 3 anos um (50\%) recebeu confirmação de diagnóstico entre 7 meses a 1 ano, e um (50\%) entre 2 a 3 anos. $\mathrm{Na}$ percepção após os 3 anos a confirmação foi de uma (100\%), após os 4 anos conforme a Tabela 5.

\section{DISCUSSÃO}

A falta de uso do aparelho auditivo prejudica o desenvolvimento de linguagem destas crianças principalmente por apresentarem uma perda de audição considerável. $\mathrm{O}$ uso da prótese auditiva proporcionaria aos sujeitos uma grande ajuda na audição, viabilizando uma melhor comunicação.

Todas as mães da pesquisa tiveram a suspeita do problema auditivo antes da criança entrar na escola. As suspeitas dos familiares para o problema auditivo foram relevantes, já que na época o Estado não disponibilizava o serviço de teste da orelhinha. 
A suspeita de deficiência auditiva é feita em $60 \%$ dos casos pelos pais e, em apenas $8 \%$, pelo profissional de saúde ${ }^{3}$.

Quanto ao motivo da suspeita do problema auditivo, a maior porcentagem foi em relação ao baruIho. Revelando coerência, pois, toda criança com audição normal apresenta reação motora ao som, ou seja, um comportamento auditivo. Pesquisa relacionada à recepção de sons, feita com lactentes entre 9 meses e 16 dias até 12 meses e 15 dias, revelam resposta de localização do som (sino) com movimentos de cabeça em $100 \%$ dos lactentes ${ }^{9}$.

Observa-se que a maioria dos entrevistados suspeita da perda auditiva no período de sete a doze meses. Pode-se supor que esta idade é responsável pelo maior percentual pelo fato do aparecimento da linguagem na criança, que no deficiente auditivo começa a ser percebido pela ausência da oralização. Na observação de pesquisadores quanto ao uso de linguagem expressiva, em lactentes entre 9 meses e 16 dias até 12 meses e 15 dias, todos apresentavam algum tipo de expressão oral, 53,8\% falando mamã/papá e $38,5 \%$ a primeira palavra ${ }^{9}$. O diagnóstico tardio ocorre devido a suspeita de deficiência auditiva apenas quando surge o atraso na fala das crianças.

O médico é o profissional a quem as mães recorrem quando percebem o problema auditivo. Os pais, quando suspeitam de qualquer alteração auditiva em sua criança, frequentemente procuram os médicos, que são os profissionais "chave" para o futuro processo de desenvolvimento de estratégias de intervenção ${ }^{10}$.

Com relação à época da procura do profissional, por falta de informação pais acabam por não pesquisar a existência de alterações auditivas e, não raramente, demoram a procurar o médico, perdendo a oportunidade de minimizar o impacto de uma deficiência auditiva, com o diagnóstico e tratamento médico corretos ${ }^{11}$.

A responsabilidade em realizar a avaliação e orientação é importante e imprescindível para os familiares terem esclarecimento sobre a deficiência auditiva. A orientação para o uso de aparelho teve maior prevalência. Obteve-se uma porcentagem significativa para a pesquisa na orientação de espera para outra reavaliação, isso sugere um desconhecimento, por parte de alguns profissionais, sobre a saúde auditiva e desenvolvimento de linguagem. Portanto, os médicos precisam ter esclarecimento sobre a questão para atender as necessidades do diagnóstico o mais precoce possível.

A detecção da deficiência auditiva deve ser rigorosamente observada por todos os profissionais da área de saúde e deve ser uma preocupação de Saúde Pública, propiciando melhoria/manutenção da qualidade de vida de nossa população infantil e na promoção da saúde auditiva ${ }^{12}$.

Relacionando a idade do diagnóstico com o tipo de deficiência auditiva da população, observa-se um período tardio de diagnóstico para o desenvolvimento de linguagem da criança. $O$ Joint Committe On Infant Hearing ( $\mathrm{JCIH})$ e o Comitê Brasileiro sobre Perdas Auditivas na Infância (CBPAI) recomendam a implantação da Triagem Auditiva Neonatal Universal (TANU), para que todo o recémnascido tenha sua audição avaliada até os três meses de idade. Nos casos de deficiência auditiva confirmada, deve haver intervenção educacional até os seis meses de idade ${ }^{13}$.

A confirmação da perda auditiva, entre o sétimo mês a um ano e após os quatro anos, corresponde as maiores porcentagens $(28,6 \%)$. Isso revela uma idade de diagnóstico além da ideal e da indicada para inferior a 6 meses de vida.

Foi constatada variação no tempo entre a suspeita e a época da detecção da perda auditiva. Ocorreu um caso de diagnóstico até seis meses. Observou-se que, algumas crianças, em que o familiar suspeitou do problema auditivo até os três meses, levaram mais de 36 meses para a confirmação.

O tempo superior a 36 meses, para a ocorrência de um diagnóstico audiológico, evidencia um período prolongado que compromete e atrasa o desenvolvimento da criança. A identificação e intervenção precoce da surdez possibilitam ao deficiente auditivo alcançar desempenho comunicativo muito próximo ao das crianças ouvintes ${ }^{14}$.

O TANU foi desenvolvido pelo $\mathrm{JCIH}$ que recomenda a realização do teste logo após o nascimento. O procedimento é muito simples. O teste da orelhinha já existe em muitos municípios do Brasil e é obrigatório por lei, mas infelizmente não muito realizado ${ }^{11}$. É fundamental o conhecimento e a valorização de todos os profissionais de saúde para que haja efetividade nos programas de triagem auditiva, tendo em vista a detecção precoce da perda auditiva por meio do teste da orelhinha ${ }^{15}$.

A prevenção e a detecção precoce de alterações no desenvolvimento infantil são práticas pouco aplicadas no Brasil, os procedimentos de triagem são utilizados por se caracterizarem como instrumentos de baixo custo, de simples aplicação e eficientes ${ }^{9}$.

No Estado do Piauí o teste da orelhinha é regulamentado por Lei n 5.370, de 09 de janeiro de 2004, e já se encontra implantado em algumas maternidades ${ }^{16}$.

\section{CONCLUSÃO}

Ressalta-se que seria importante a educação em saúde sobre orientações acerca do desenvol- 
vimento auditivo das crianças, antes que as mães saíssem das maternidades com os recém-nascidos. Embora as mães não tivessem oportunidades de realizar o exame, ficariam mais atentas à audição da criança e talvez a suspeita e o diagnóstico ocorressem em tempo mais precoce possível. Assim, neste contexto tornam-se relevantes as informações coletadas na pesquisa realizada.

Podem-se observar os seguintes aspectos que contribuíram para um diagnóstico tardio da perda auditiva:

A presença da perda auditiva foi suspeitada somente quando o familiar observou atraso de fala, e isto não se constitui em atitude satisfatória;

A maior porcentagem de suspeita ocorreu quando a criança estava na idade de 7 a 12 meses, pois, nesta época os familiares observaram ausência de oralização;

Ocorreu demora na busca do profissional da saúde, e a orientação deste para esperar foi relevante no diagnóstico;

A identificação foi tardia ao serem considerados o tipo e o grau de perda auditiva apresentados pelas crianças assistidas na APADA, que suscitam sinais nítidos, no mínimo, para o profissional atento às respostas da criança frente aos sons.

Mediante a pesquisa, sugere-se alertar os profissionais e afins envolvidos da área de saúde para observar os fatores relevante sobre audição e das alterações auditivas.

\section{AGRADECIMENTOS}

Aos Professores João Batista Teles; Laureni Dantas de França; Cecília Paz; Edna Ferraz Moura e Fernando José Pedrini. Aos pais Ernesto Mendes de Sousa Caldas e Maria de Fátima Araújo Mendes; Carlos Alves de Araújo e Nilena Maria de Brito Monteiro Araújo. Às filhas de Nilena Carla Monteiro Araújo Arêa Leão, Laís Monteiro Araújo Campos Arêa Leão e Maria Antônia Monteiro Araújo Campos Arêa Leão. À irmã de Janaína Mendes de Sousa Caldas, Natália Mendes de Sousa Caldas. Às mães dos alunos assistidos e a APADA e, pela oportunidade do desenvolvimento da pesquisa e por tamanha receptividade.

\begin{abstract}
Purpose: to analyze and characterize children suspected by the family of hearing loss attended at Associação de Pais e Amigos do Deficiente Auditivo (APADA) in Teresina, PI. Methods: a descriptive study was carried out at PFHIA from September to October 2006. Form were filled out by 14 mothers and/or the responsible adult to find the following variables: identify the family member who suspected hearing loss in the child; the reason which led to the suspicion; the age of the child when hearing loss was detected by the family member; the attitude of the family after discovering the hearing loss; the age of the child when professional help was obtained and the orientation received; the time lapse from suspicion of hearing loss by the family to professional confirmation. Results: the data shows that hearing loss was suspected by the mothers in $86 \%$. The reason for the suspicion was no reaction to sound from the child $(71 \%)$. 36\% of the families noticed the problem at the age of 7 to 12 months, $79 \%$ looked for medical help as their first attitude, 36\% looked for a health professional before 1 year of age, $29 \%$ received the health orientation to use hearing aids and had the confirmation of hearing loss from 7 months to 1 year of age or after the age of 4 . Conclusion: the diagnosis of hearing loss was late and discovered by members of the family and health professionals and was not related to newborn hearing screening programs.
\end{abstract}

KEYWORDS: Hearing; Hearing Loss; Language; Diagnosis

\section{REFERÊNCIAS}

1. Gatto Cl, Tochetto TM. Deficiência auditiva infantil: implicações e soluções Rev CEFAC. 2007; 9(1): 110-15. dx.doi.org/S1516-18462007000100014

2. Vieira ABC, Macedo LR. O diagnóstico da perda auditiva na infância. Pediatria. 2007; 29(1):43-9.
3. Lamprecht A. Evozierte otoakustische emissionen bel normallhoerenden und schwerhorigen erwachsenen und kindern. LaryngoRhino-Otho. 1991; 70:1-4.

4. Gordo A. Distúrbios auditivos. In: Sacaloski M, Alavasi E, Guerra GR. Fonoaudiologia na escola. São Paulo: Lovoise; 2000. p.181-98. 
5. Borges CAB, Moreira LMO, Pena GM, Fernandes $\mathrm{FR}$, Borges $\mathrm{BCB}$, Otani $\mathrm{BH}$. Triagem auditiva neonatal universal. Arq Int Otorrinolaringol. 2006; 10(1):28-34.

6. Pupo, AC, Balieiro CR, Figueiredo RSL. Estudo retrospectivo de crianças e jovens com deficiência auditiva: caracterização das etiologias e quadro audiológico. Rev. CEFAC. 2008; 10(1):84-91. dx.doi.org/S1516-18462008000100012

7. American Speech-Language-Hearing Association [serial on the Internet]. Effects of hearing loss on development. [updated 2007; cited 2008 march 23]. Available from: URL: http://www.asha.org/public/ hearing/disorders/effects.htm

8. Carvalho FL, Alencar MAPB. Conhecimento dos pediatras sobre a atuação do profissional fonoaudiólogo [monografia]. Teresina (PI): Faculdade de Ciências, Humanas e Tecnológicas do Piauí; 2006.

9. Lima, MCMP, Barbarini, GC, Gagliardo, HGRG, Arnais MAO, Gonçalves VMG. Observação do desenvolvimento de linguagem e funções auditiva e visual em lactentes. Rev Saúde Pública. 2004; 38(1):106-12.

10. Lichtig I, Cárnio MS, Couto MIV. Surdez e reabilitação baseada na comunidade. In: Andrade CRF, Marcondes E. Fonoaudiologia em pediatria. São Paulo: Sarvier; 2003. p.113-9.
11. Sousa LCA. Campanha nacional da audição. [serial on the internet]. 2006 [citado 2006 set 10]. Disponível em: URL: http://www.saudeauditiva.org. br/artigos/artigos_detalhe. asp?id=1

12. Harrison M, Roush J, Wallace J. Trends in age of identification and intervention in infants with hearing loss. Ear Hear. 2003; 24(1):89-95.

13. Joint Committee on Infant Hearing. Year 2000. Position statement: principles and guidelines for early hearing detection and intervention programs. Am J Audiol. 2000; 9(1):9-29.

14. Colunga JCM, Méndez JCA, Villarreal JMC, Zapico MJA, Estrada CM, Alvarez MLF. Despistage de La hipoacusia neonatal: resultados después de 3 años de iniciar nuestro programa. Acta Otorrinolaringol Esp. 2005; 55:55-8.

15. Hilú MRPB, Zeigelboim BS. O conhecimento, a valorização da triagem auditiva neonatal e a intervenção precoce da perda auditiva. Rev CEFAC. 2007; 9(4):563-70. dx.doi.org/ S1516-18462007000400017

16. Piauí. Assembléia Legislativa. Lei no 5.370, de 09 de janeiro de 2004. Dispõe sobre a obrigatoriedade da realização do exame de Emissões Evocadas Otoacústicas e dá outras providências.

RECEBIDO EM: 02/07/2008

ACEITO EM: 23/03/2009

Endereço para correspondência:

Janaína Mendes de Sousa Caldas

Rua Mato Grosso, 112 ap. 503

Teresina - PI

CEP: $64014-150$

E-mail: jana_caldas@hotmail.com 